\title{
Future of Monte Carlo simulations of atmospheric showers
}

\author{
Tanguy Pierog ${ }^{1, \text { a }}$, Ralph Engel ${ }^{1}$, Dieter Heck ${ }^{1}$, and Gevorg Poghosyan ${ }^{2}$ \\ ${ }^{1}$ Karlsruhe Institute of Technology (KIT), IKP, 76021 Karlsruhe, Germany \\ ${ }^{2}$ Simulation Lab E\&A Particles, SCC, 76021 Karlsruhe, Germany
}

\begin{abstract}
In 2013, the air shower simulation model CORSIKA had a major release opening new windows in term of uncertainty due to hadronic interaction models and of simulation time. On the one hand, the two hadronic models EPOS and QGSJETII were updated taking into account new LHC data. As a consequence the uncertainties in air shower observables were reduced by about a factor of 2 at the highest energies. On the second hand, two new possibilites of running CORSIKA were introduced: either in a parallel mode on big CPU clusters allowing the simulation of unthinned showers in a reasonable time, or using cascade equations to reduce the simulation time by about of factor of 10 on a single CPU. All these improvements will be presented.
\end{abstract}

\section{Introduction}

The experimental method of studying ultra-high energy cosmic rays is an indirect one. Typically, one investigates various characteristics of extensive air showers (EAS), a huge nuclear-electromagnetic cascade induced by a primary particle in the atmosphere, and uses the obtained information to infer the properties of the original particle, its energy, type, direction, etc.. Hence, the reliability of ultra-high energy cosmic ray analyzes depends on the use of proper theoretical and phenomenological descriptions of the cascade processes.

The most natural way to predict atmospheric particle cascading in detail seems to be a direct Monte Carlo (MC) simulation of EAS development, like it is done, for example, in the CORSIKA program [1]. As very large computation times are required at ultra-high energy, a parallelization of the sub-showers of the cascade on big CPU clusters is now possible. An alternative procedure was developed to describe EAS development numerically, based on the solution of the corresponding cascade equations (CE). Combining this with an explicit MC simulation of the most energetic part of an EAS allows us to obtain accurate results both for average EAS characteristics and for their fluctuations in the CONEX program [2]. Combining the two programs not only a complete $3 \mathrm{D}$ simulation can be achieved with a reduced computation time but new analysis possibilities are open.

One fundamental ingredient of the MC process is the hadronic interaction model which generates the hadronic cascade at the origin of the electromagnetic cascade. Since 2009, the Large Hadron Collider (LHC) provides a lot of very precise data which have been used to improve two of the hadronic models used for air shower simulations: EPOS [3] and QGSJETII [4]. Despite the extrapolation still needed to reach the highest energy cosmic-rays (Fig. 1), a significant improvment has been made in model predictions.

In this article, we will discuss changes in the hadronic model predictions after LHC data and their consequences

a e-mail: tanguy ·pierog@kit.edu

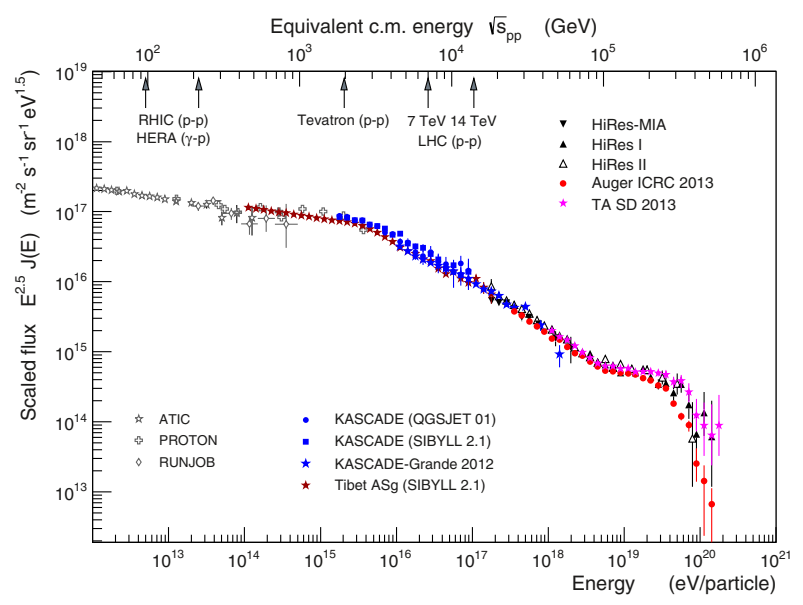

Figure 1. Flux of cosmic rays arriving at Earth rescaled by the energy to the power 2.5. Data references are given in [5].

on air shower observables. In the first section, we will compare the results of the newly available hadronic interaction models, EPOS LHC and QGSJETII-04 with LHC data for the main observables relevant for air shower development. Then the main new options available in the last release of CORSIKA v7.4x and CONEX v4.37 will be reviewed. Finally using detailed Monte Carlo simulations done with these air shower simulation models, the new predictions for $\mathrm{X}_{\max }$ and for the number of muons and missing energy will be presented.

\section{Hadronic interaction models and LHC data}

To qualitatively describe the dependence of shower development based on some basic parameters of particle interaction, decay and production, a very simple toy model can be used. Although initially developed for electromagnetic (EM) showers [6] it can also be applied to hadronic showers [7].

It is clear that such a toy-model only gives a very much over-simplified account of air shower physics. However, the model allows us to qualitatively understand

This is an Open Access article distributed under the terms of the Creative Commons Attribution License 4.0, which permits unrestricted use, distribution, and reproduction in any medium, provided the original work is properly cited. 
the dependence of many air shower observables on the characteristics of hadronic particle production. Accordingly the parameters of hadron production which are most important for air shower development are the cross section (or mean free path), the multiplicity of secondary particles of high energy, and the production ratio of neutral to charged particles. Until the start of LHC, these parameters were not well constrained by particle production measurements at accelerators. As a consequence, depending on the assumptions of how to extrapolate existing accelerator data, the predictions of hadronic interaction models differ considerably.

There are several hadronic interaction models commonly used to simulate air showers. Here we will focus on the two high energy models which were updated to take into account LHC data at 7 TeV: QGSJETII-03 [4,8] changed into QGSJETII-04 [9] and EPOS 1.99 [3,10] was replaced by EPOS LHC (V3400), both available in CORSIKA V7.4X [1]. There is no major change in these models but in addition to some technical improvements, some parameters were changed to reproduce TOTEM [11] cross sections. Both are based on Gribov-Regge multiple scattering, perturbative QCD and string fragmentation. The former versions reproduce accelerator data and even first LHC data reasonably well ([12] and Figs. 3 and 4) but predict different extrapolations above $E_{\mathrm{cms}} \sim 1.8 \mathrm{TeV}$ $\left(E_{\mathrm{lab}} \sim 10^{15} \mathrm{eV}\right)$ that lead to very different results at high energy $[13,14]$ which can be improved using LHC data.

\subsection{Cross section}

As shown in [7], the cross section is very important for the development of air showers and in particular for the depth of shower maximum. As a consequence, the number of electromagnetic particles at ground level is strongly correlated to this observable (if the shower maximum is closer to ground, the number of particles is higher).

The total cross section of proton-proton scattering is usually used as an input to fix basic parameters in all hadronic interaction models. Therefore it is very well described by all the models at low energy, where data exist [15]. And then it diverges above $2 \mathrm{TeV}$ center-of-mass (cms) energy because of different model assumptions. As shown on Fig. 2 the new point measured by the TOTEM experiment at $7 \mathrm{TeV}$ reduces the difference between the models by a factor of 5 (50 to $10 \mathrm{mb})$. In all the figures EPOS LHC is represented by a full (blue) line, QGSJETII-04 by a dotted (red) line, EPOS 1.99 by a dashed (black) line and QGSJETII-03 by a dashed-dotted (green) line.

\subsection{Multiplicity}

According to [7], the multiplicity plays a similar kind of role as the cross section, but with a weaker dependence $(\log )$. On the other hand, the predictions from the models had much larger differences for the multiplicity compared to the cross section. As shown in Fig. 3 left-hand side, the particle density at mid-rapidity is well reproduced by all the models up to $2 \mathrm{TeV}$ where Tevatron data [17] constrain the results, but at the highest energies in $\pi$-air,

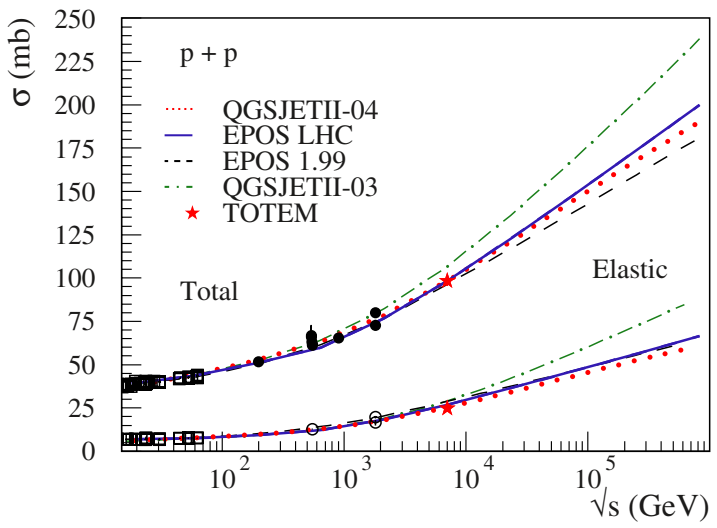

Figure 2. Total and elastic p-p cross sections calculated with EPOS LHC (full line), QGSJETII-04 (dotted line), EPOS 1.99 (dashed line) and QGSJETII-03 (dashed-dotted line). Points are data from [16] and the stars are the LHC measurements by the TOTEM experiment [11].

the difference can be as high as a factor of 10 (Fig. 3 right-hand side). After re-tuning at $7 \mathrm{TeV}$ to be compatible with CMS data [18] or ALICE data [19], in Fig. 4, the difference, even at high energy in $\pi$-air, is less than a factor of 2. On the right-hand side of Fig. 4, we can see that not only the averaged multiplicity had been changed after re-tuning, but the fluctuations are now very similar for QGSJETII-04 and EPOS LHC. This will be important for the fluctuations of the air shower maximum.

So, for both cross section and multiplicity, when the models are constrained by LHC data up to $7 \mathrm{TeV}$, the extrapolation to the highest energy is not so different any more. This will have a strong impact on $\mathrm{X}_{\max }$ uncertainty in air shower simulations.

\subsection{Baryon production}

Another important observable for EAS is the number of muons reaching the ground. Using again [7], it has been shown in [20] that the number of muons in EAS depends on the ratio $R$ between all particle types except $\pi^{0}$ and the total number of particles and hence is sensitive to the number of (anti)baryons produced in the hadronic interactions. So it is important to check the production of such particles in LHC data.

Both ALICE [21] and CMS [22] experiments published very nice results on identified spectra used to constrain models used for air shower simulations. As shown in Fig. 5, these data help a lot to reduce the differences between the models especially because they resolve an ambiguity on the phase space used to produce the anti-proton over pion ratio as a function of the number of charged particles with Tevatron data at $1.8 \mathrm{TeV}$. LHC data are much better defined and can be used to constrain the production of baryon pairs at mid-rapidity (largely dominated by string fragmentation).

It is important to notice that not only (anti)baryons enter into the definition of the ratio $R$. All particles which do not decay into an electromagnetic particle can play a similar role and keep the energy of the shower into the hadronic channel to produce muons. For instance 

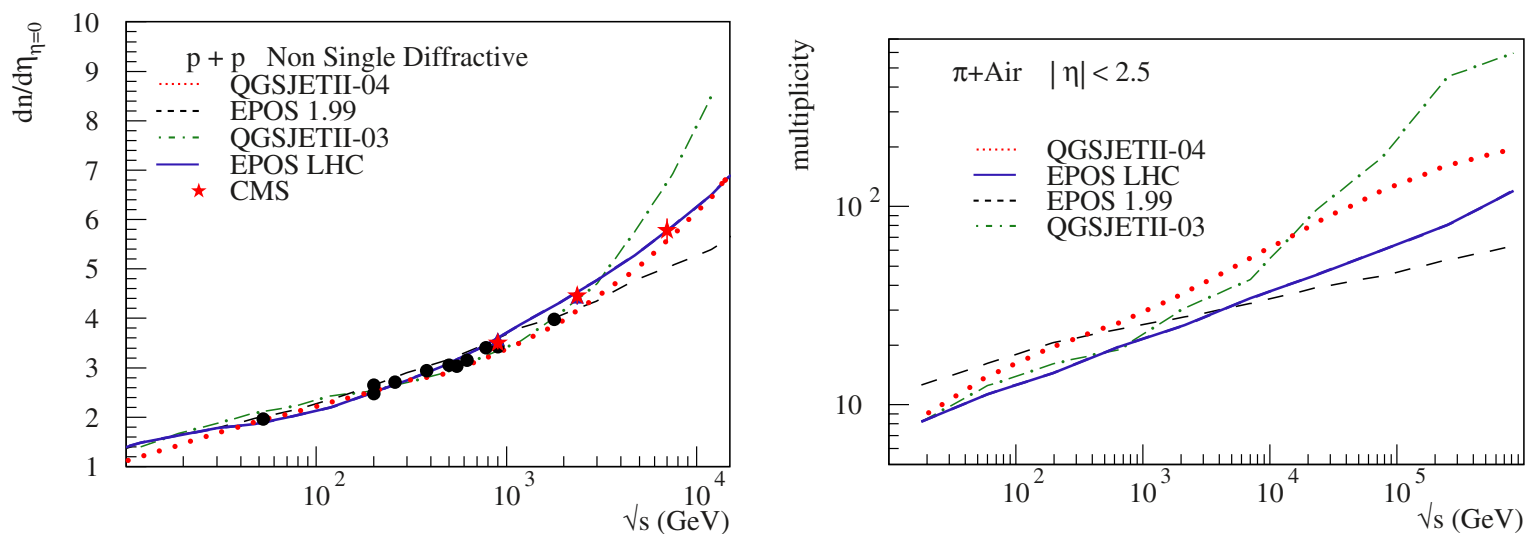

Figure 3. Particle density at $\eta=0$ for non single diffractive events (NSD) (left-hand side) and multiplicity for $|\eta|<2.5$ of $\pi$-air collisions (right-hand side) as a function of center of mass energy. Simulations are done with EPOS LHC (full line), QGSJETII-04 (dotted line), EPOS 1.99 (dashed line) and QGSJETII-03 (dashed-dotted line). Points are data from old experiments and red stars are from CMS experiment [18].
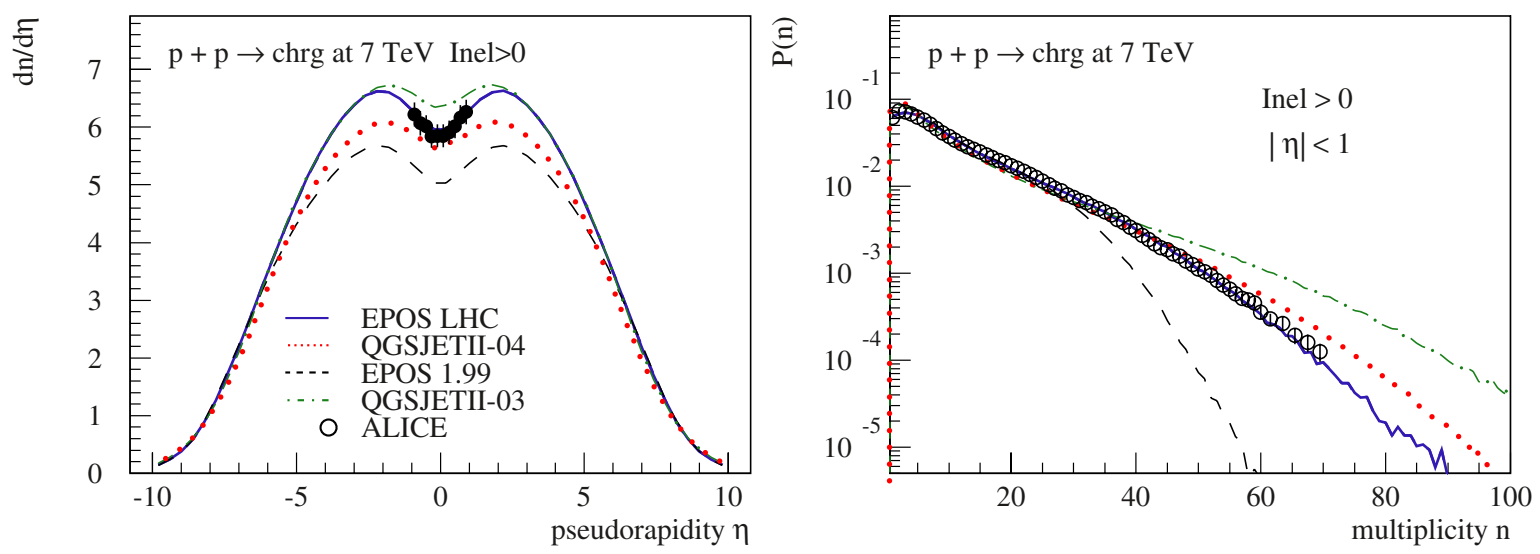

Figure 4. Pseudorapidity distribution $d N / d \eta$ for events with at least one charged particle with $|\eta|<1$ (left-hand side) and corresponding multiplicity distribution (right-hand side) for p-p interactions at $7 \mathrm{TeV}$. Simulations with EPOS LHC (full line), QGSJETII-04 (dotted line), EPOS 1.99 (dashed line) and QGSJETII-03 (dashed-dotted line) are compared to data points from the ALICE experiment [19].
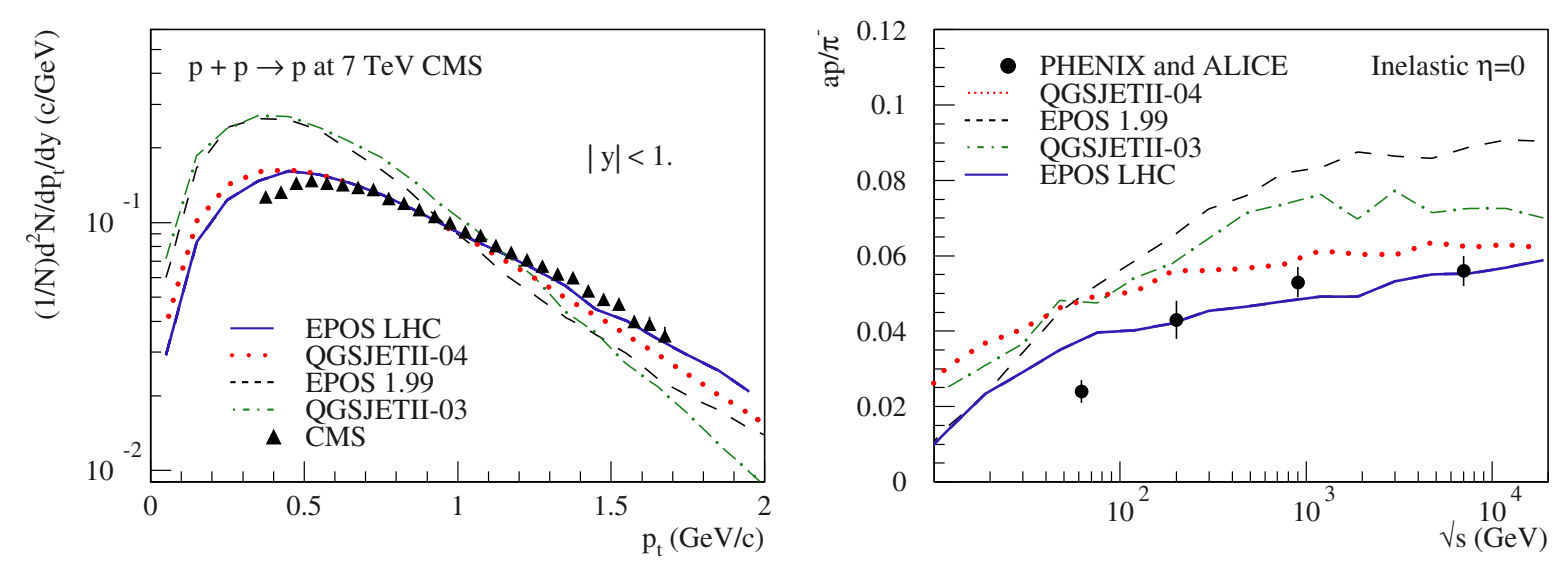

Figure 5. Transverse momentum distribution for $|y|<1$ for non single diffractive (NSD) p-p scattering at 7 TeV (left-hand side) and anti-proton over pion ratio at $y=0$ for p-p collisions (right-hand side) as a function of center of mass energy. Simulations are done with EPOS LHC (full line), QGSJETII-04 (dotted line), EPOS 1.99 (dashed line) and QGSJETII-03 (dashed-dotted line). Points are data from ALICE and PHENIX [21] experiments and triangles are from CMS experiment [22]. 

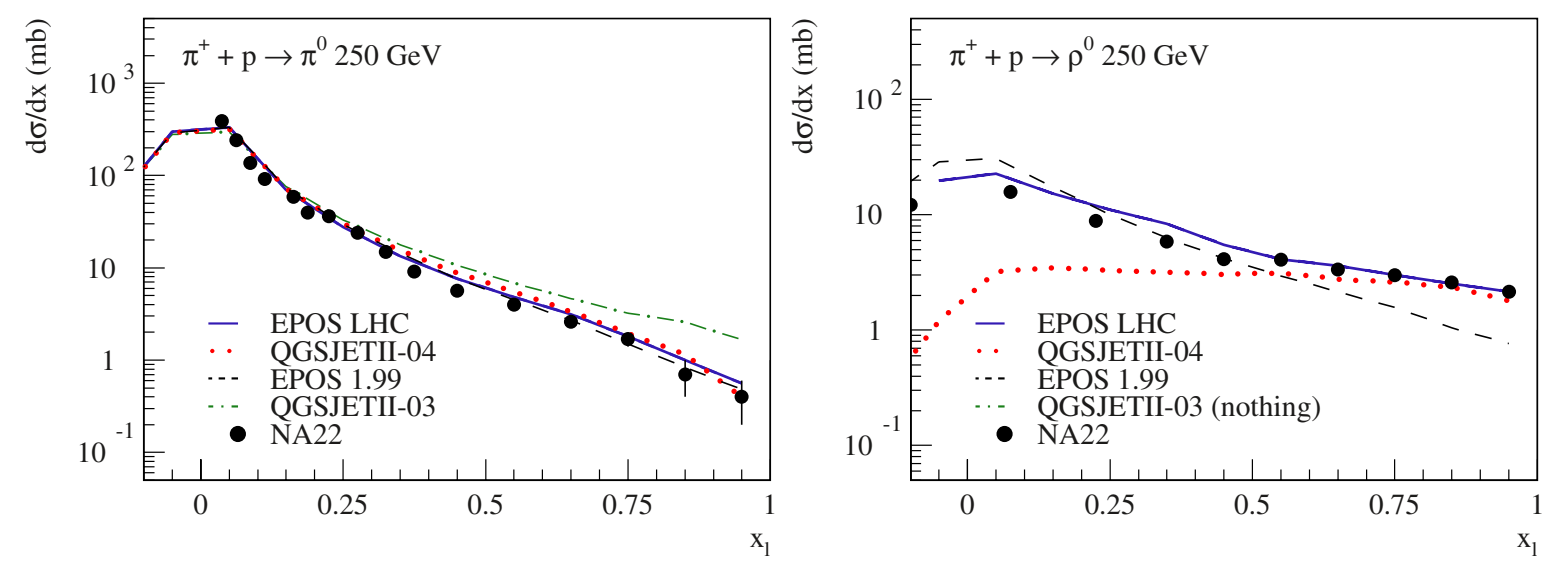

Figure 6. $\pi^{0}$ longitudinal momentum fraction distribution (left-hand side) and $\rho^{0}$ longitudinal momentum fraction distribution (righthand side) for $\pi$-p interactions at $250 \mathrm{GeV}$. Simulations with EPOS LHC (full line), QGSJETII-04 (dotted line), EPOS 1.99 (dashed line) and QGSJETII-03 (dashed-dotted line) are compared to data points from NA22 experiment [23,24].

in QGSJETII-04 the newly introduced $\rho^{0}$ resonance as excited state of the pion remnant in pion interactions has a very strong influence on the muon production. Since forward $\pi^{0} \mathrm{~s}$, transferring a lot of energy in the electromagnetic channel, are replaced by particles which decay into charged pions, the energy is kept in the hadronic channel. This is clearly illustrated by the Fig. 6 where we can see that QGSJETII-04 reproduce nicely both $\pi^{0}$ and $\rho^{0}$ forward spectra while QGSJETII-03 producing no $\rho^{0}$ had too hard $\pi^{0}$ spectra. This effect was already in EPOS 1.99, being one source of difference between the 2 models. EPOS 1.99 model seems to underestimate $\rho^{0}$ production but this is due to a wrong mixing with $\omega$ resonance and, in fact, the sum of the 2 is correct. It has been corrected in EPOS LHC. On the other hand, in EPOS 1.99 another process producing forward (anti)baryons was missing at high energy and has been added in EPOS LHC. As a consequence the reduced rate of (anti)baryon production at mid-rapidity is compensated by more forward (anti)baryon production which is even more important for muon production. Unfortunately there are very little data to constrain this production channel especially in collider experiments. NA61 [25] and LHCf [26] experiments may help to constrain this process in the future.

\section{EAS simulations}

\subsection{CORSIKA v7.4x}

CORSIKA 7.4x are available since April 2013 including post-LHC hadronic models EPOS LHC and QGSJETII04. The main new option is the possibility to replace the thinning procedure by numerical cascade equations as implemented in CONEX. The detailed process described here and was first implemented in the SENECA model [27].

\subsubsection{Hybrid simulations}

In order to have the best of CONEX and CORSIKA in one single program, we are using the method outlined in

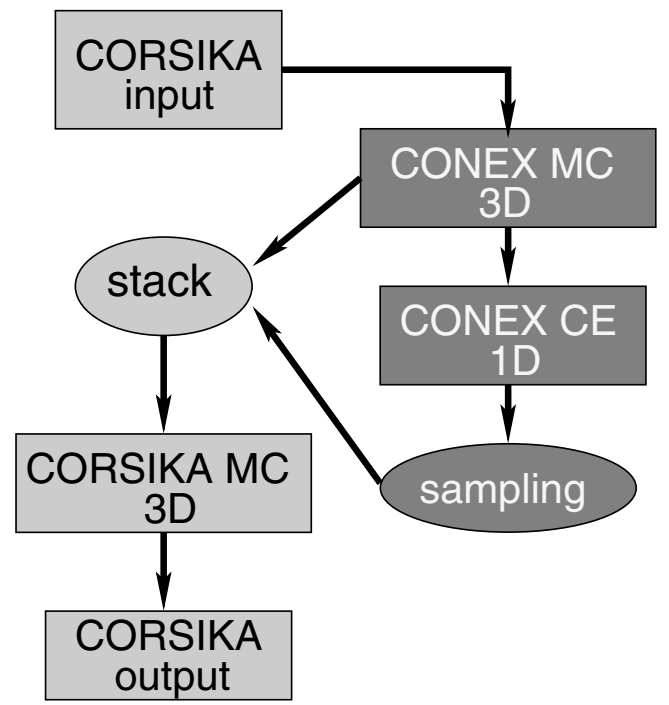

Figure 7. Implementation of CONEX in CORSIKA.

Fig. 7. The CORSIKA installation scheme and steering files are used to set the simulation parameters. Then, internally, these parameters are transferred to CONEX to start the MC simulation with the given primary energy. Depending on their energy, the secondary particles stay either in CONEX MC if $E>E_{\mathrm{thr}}$, or go into the CORSIKA stack if $E<E_{\text {low }}$, or are used as source for 1-dimensional $\mathrm{CE}$ in the energy range in between. When no more particles with $E>E_{\mathrm{thr}}$ are stored on the CONEX stack, the $\mathrm{CE}$ are solved down to $E_{\text {low. The solution of }}$ the $\mathrm{CE}$ can be sampled into individual particles saved on the CORSIKA stack. At this point, a weight can be attributed to these particles to reduce the simulation time. Finally all these particles with $E<E_{\text {low }}$ stored in the stack are tracked in CORSIKA as usual in a 3-dimensional space until they reach the observation level where they are stored in the chosen output file. As a result, simulations can be done either in 1D (only the longitudinal profile) or in 3D (lateral distribution function (LDF)) or even 3D only for the muons depending on keyword used in the steering file (automatic threshold management). For an equivalent precision level, a minimum gain factor 


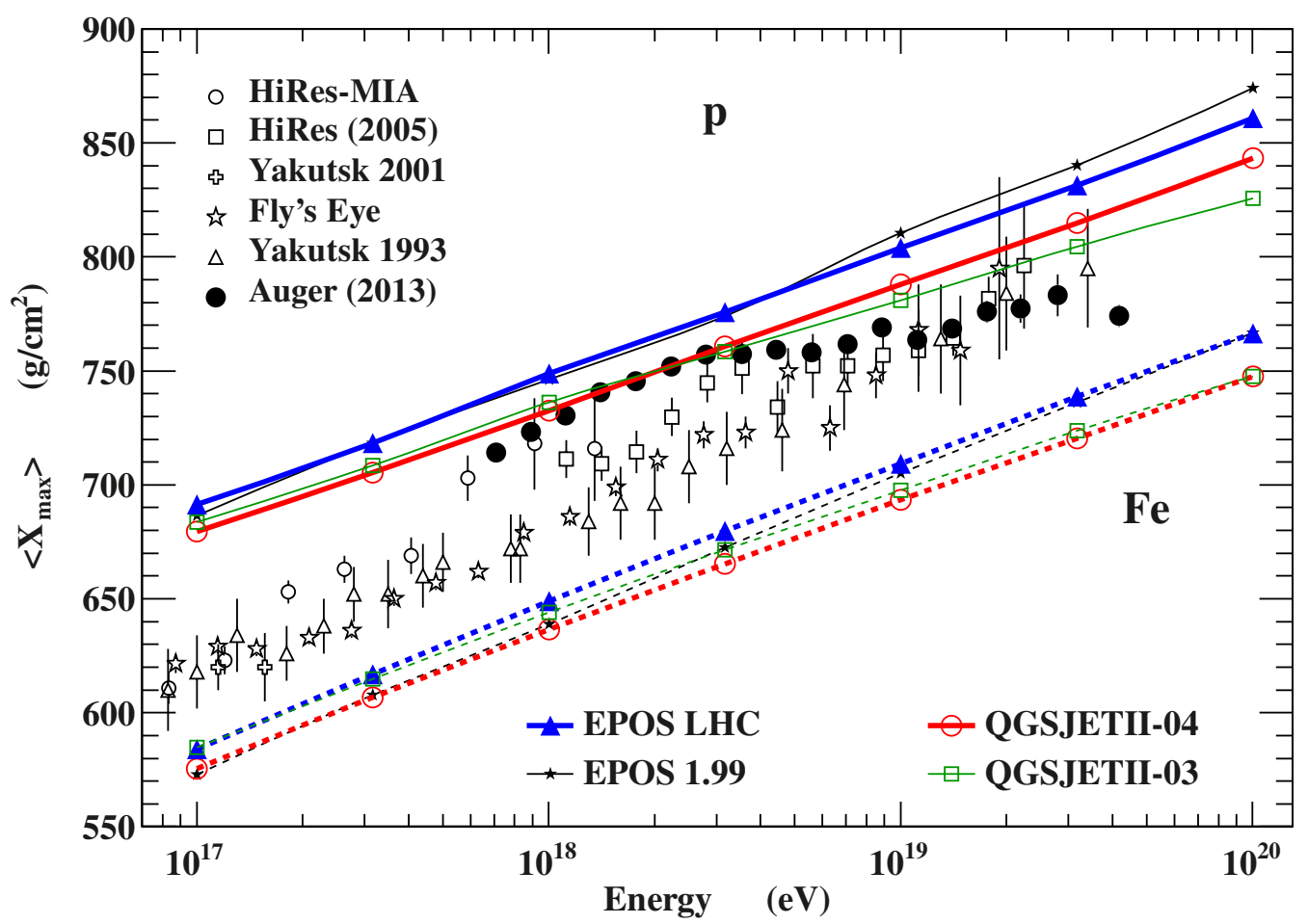

Figure 8. Mean $X_{\max }$ for proton and iron induced showers are shown as a function of the primary energy for different high-energy hadronic interaction models, full lines for proton and dashed lines for iron with full triangles for EPOS LHC, open squares for QGSJETII-03, open circles for QGSJETII-04, and full stars for EPOS 1.99. Refs. to the data can be found in [32] and [33].

of 5 to 10 in time can be expected using this method instead of standard thinning. When only the longitudinal development is needed, the simulation time is reduced to a couple of minutes for all primary energies.

Using the new option, a new approach is possible to analyze air shower data. Until now, only statistical analyzes were possible because of the large shower-byshower fluctuations and the large computation time. But since the same first sequence of hadronic interactions are realized independly of the selected option (1D or 3D full $\mathrm{MC})$ for a given random seed, the fluctuations induced by the low energy $\mathrm{MC}$ are really limited (not affecting $X_{\max }$ for instance). Using hybrid simulation (CORSIKA + CONEX) together with the new hybrid detectors (fluorescence+surface (PAO [28], Telescope Array [29]), radio+surface (PAO, KASCADE [30]), ...) it is possible to study data shower-by-shower.

\subsubsection{Parallelization}

Another new option available in CORSIKA $7.4 \mathrm{x}$ is the possibility to run unthinned shower using massive parallelization with Message Passing Interface (MPI) management.

To overcome the extreme long computing time for a single shower induced by an ultra-high energy primary particle the simulation task has to be split into many jobs which are treated in parallel on many cores of a computer cluster. In our approach the results of such parallel simulations are controled in a unique way by seeds for the random number generator given by the user. It is now possible to collect all particles above a user-defined threshold in an external file, which can be used to run all the sub-showers induced by these particles on different CPU's. If the seeds are well defined for each sub-shower, it is possible to reproduce the same shower under different technical conditions of the available computing resources. A challenge is the merging and handling of the huge final file containing all particles which arrive at ground.

To help book-keeping and for a better management of all the sub-showers on large clusters, there is the possibility to use CORSIKA as a subroutine of a master program which can distribute all the sub-showers on different CPU using MPI protocol. In that case, there is no need for an external file to save temporary stack.

As a side effect, an option has been added to introduce a particle or a list of particles anywhere in space independently of the shower axis. This can be used to study artificial showers having special features.

\subsection{CONEX v4.37}

The corresponding version of CONEX linked to ROOT has been released with version number 4.37 for 1D use only. New hadronic models EPOS LHC and QGSJETII04 are available and the low energy model GHEISHA has been replaced by URQMD 1.3 [31] as used in CORSIKA.

\subsection{Results}

In the following EAS simulation results using EPOS LHC and QGSJETII-04 are presented and compared to former results using QGSJETII-03 and EPOS 1.99. 


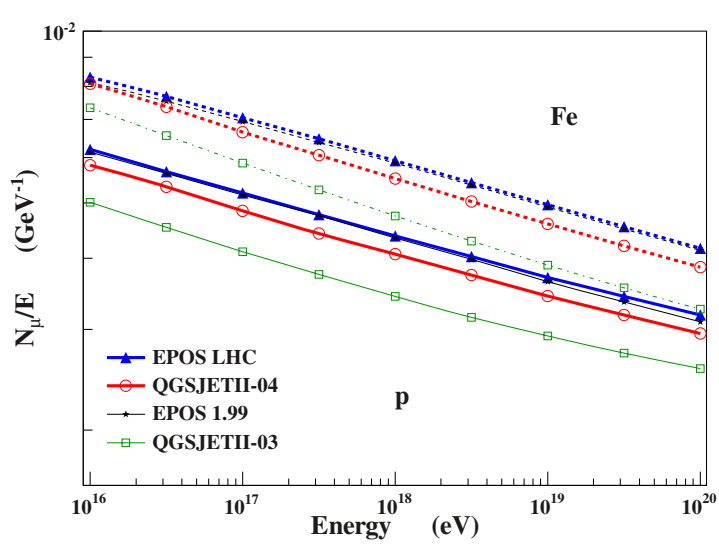

Figure 9. Mean number of muons at ground divided by the primary energy for proton and iron induced showers are shown as a function of the primary energy for different high-energy hadronic interaction models, full lines for proton and dashed lines for iron with full triangles for EPOS LHC, open squares for QGSJETII-03, open circles for QGSJETII-04, and full stars for EPOS 1.99.

As shown in Fig. 8, the mean depth of shower maximum, $\mathrm{X}_{\max }$, for proton and iron induced showers simulated with CONEX is still different for EPOS LHC and QGSJETII-04. But now the elongation rate (the slope of the mean $\mathrm{X}_{\max }$ as function of the primary energy) is the same in both cases while EPOS 1.99 had an elongation rate larger than QGSJETII-03. The difference between the 2 models is a constant shift of about $20 \mathrm{~g} / \mathrm{cm}^{2}$ (close to the experimental systematic error in PAO [32]) while before the difference was increasing up to $50 \mathrm{~g} / \mathrm{cm}^{2}$ at the highest energies

This is very important to study the primary cosmic ray composition. If the models converge to a similar elongation rate, it will allow us to have a more precise idea on possible changes in composition at the "ankle" for instance where the PAO measured a break in the elongation rate of the data.

Concerning the number of muons at ground (for $40^{\circ}$ inclined shower at the height of $1500 \mathrm{~m}$ ), the difference between the new QGSJETII-04 and the old QGSJETII03 is even more impressive. We can see on Fig. 9 that QGSJETII-04 predicts now about the same number of muons as EPOS 1.99 which is about $20 \%$ more than QGSJETII-03. It is due to the change in baryon, strangeness and mostly resonance production as described in Sect. 2.3. Concerning the predictions of EPOS LHC, the number of muons is very similar to the one in EPOS 1.99 because of the leading baryon production compensating the reduction of (anti)aryon production at mid-rapidity. So, even if the number of muons is much more similar now for the two most recent hadronic models, there is still an uncertainty of about $10 \%$ and furthermore the energy spectrum of the muons at ground is different between the models. As a consequence the mean decay length is different and the signal as a function of the zenith angle will behave differently. On Fig. 10, we plot the muon signal at ground as measured by PAO experiment (at $1000 \mathrm{~m}$ from the shower core for an primary energy of $10^{19} \mathrm{eV}$ and ground height at $1500 \mathrm{~m}$ ) divided by the

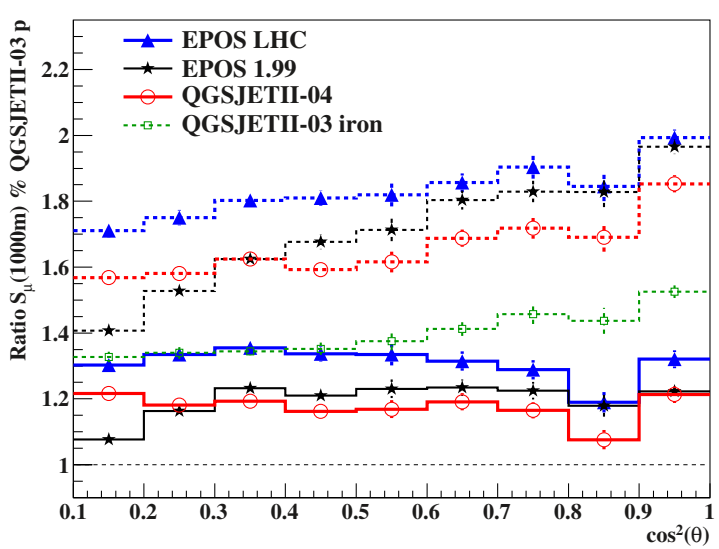

Figure 10. Ratio of muon signal at $1000 \mathrm{~m}$ at ground as measured by PAO with respect to QGSJETII-03 proton showers for proton and iron induced showers at $10^{19} \mathrm{eV}$ as a function of the zenith angle $\left(\cos ^{2} \theta\right)$. Predictions of different high-energy hadronic interaction models: full lines for proton and dashed lines for iron with full stars for EPOS LHC, open squares for QGSJETII-03, open circles for QGSJETII-04, and full triangles for the results of EPOS 1.99 .

one of QGSJETII-03. We can see that QGSJETII-04 predictions are larger but parallel to the one of QGSJETII03. Concerning the predictions of EPOS LHC, the number of muons is very similar to the one in EPOS 1.99 for vertical showers but the attenuation length being larger (closer to QGSJETII-04 but still with a lower mean energy for muons) the difference at large angle is as large as $20 \%$. This is probably due to the leading baryon production compensating the reduction of (anti)baryon production at mid-rapidity but for a different phase space so with a different energy.

A consequence of the larger mean energy of the muons in EAS simulated with QGSJETII-04 is that the missing energy is larger for showers simulated with this model even if the total number of muons is larger in EPOS LHC. EPOS LHC results are very similar to those of EPOS 1.99. The factor to correct for the missing energy in fluorescence based telescopes is presented on Fig. 11. The difference between QGSJETII-04 and the previous version QGSJETII-03 frequently used as reference in the past is less than $3 \%$ but it has a direct influence on the energy scale considered to compare simulations and data. It should not be neglected.

\section{Summary}

Using recent $\mathrm{LHC}$ data at $7 \mathrm{TeV}$ it is possible to reduce the uncertainty in the extrapolation of the hadronic interaction models used for EAS simulations. Using preand post-LHC versions of the QGSJETII and EPOS models included in the last release of CORSIKA v7.4x and CONEX v4.37, it has been shown that the difference in multiplicity between these models has been reduced by a factor of 5 at the highest energy, resulting in a very similar elongation rate. There is still a systematic shift in $X_{\max }$ of about $20 \mathrm{~g} / \mathrm{cm}^{2}$ due to remaining differences in the multiplicity (and elasticity) of the models. This 


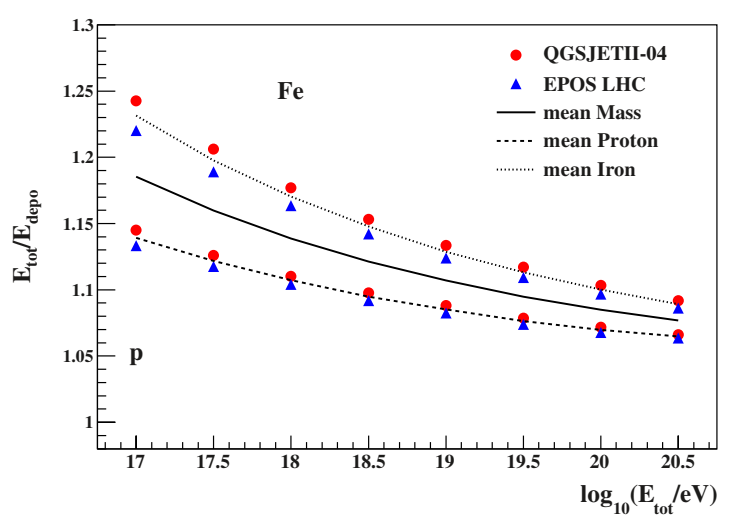

Figure 11. Inverse of missing energy factor for proton and iron induced showers as a function of the primary energy. Predictions of different high-energy hadronic interaction models are shown with triangles for EPOS LHC and circles for QGSJETII-04. Lines are averages between models.

uncertainty is comparable to the experimental uncertainty in the measurement of $\mathrm{X}_{\max }$. As a consequence the interpretation of the data using post-LHC data will be more reliable especially concerning the possible change in mass composition with energy as summarized in [34].

For the number of muons, the ratio between particles producing hadronic sub-showers and the total number of particles is very important. LHC data are important to constrain (anti)baryon and strangeness production at midrapidity. Lower energy data of fixed target experiment are also important to measure forward production of $\pi^{0}$ for instance. Taking into account both aspects, the new versions of the QGSJETII and EPOS models predict very similar results close to EPOS 1.99 model (less than $10 \%$ difference) but with a flatter attenuation length.

Compared to QGSJETII-03, using both post-LHC hadronic models to interpret cosmic ray data will result in a slightly lighter composition around the "knee" [35] and heavier composition above the "ankle" [36].

\section{References}

[1] D. Heck, J. Knapp, J. Capdevielle, G. Schatz, T. Thouw (https://web.ikp.kit.edu/corsika/), Wissenschaftliche Berichte, Forschungszentrum Karlsruhe FZKA 6019 (1998)

[2] T. Bergmann et al., Astropart. Phys. 26, 420 (2007), astro-ph/0606564

[3] K. Werner, F.M. Liu, T. Pierog, Phys. Rev. C74, 044902 (2006), hep-ph/0506232

[4] S. Ostapchenko, Phys. Rev. D74, 014026 (2006), hep-ph/0505259

[5] R. Engel, D. Heck, T. Pierog, Ann. Rev. Nucl. Part. Sci. 61, 467 (2011)

[6] W. Heitler, Quantum Theory of Radiation (Oxford University Press, Oxford, 1944), 2nd edition
[7] J. Matthews, Astropart. Phys. 22, 387 (2005)

[8] S. Ostapchenko, Phys. Lett. B636, 40 (2006), hep-ph/0602139

[9] S. Ostapchenko, Phys. Rev. D83, 014018 (2011), 1010.1869

[10] T. Pierog, K. Werner, Nucl. Phys. Proc. Suppl. 196, 102 (2009), 0905.1198

[11] T. Csörgö et al. (TOTEM Collaboration), Prog. Theor. Phys. Suppl. 193, 180 (2012), 1204.5689

[12] D. d'Enterria, R. Engel, T. Pierog, S. Ostapchenko, K. Werner, Astropart. Phys. 35, 98 (2011), 1101.5596

[13] J. Knapp, D. Heck, S.J. Sciutto, M.T. Dova, M. Risse, Astropart. Phys. 19, 77 (2003), astro-ph/0206414

[14] R. Engel, H. Rebel, Acta Phys. Polon. B35, 321 (2004)

[15] H. Jung et al. (2009), 0903.3861

[16] C. Caso et al. (Particle Data Group), Eur. Phys. J. C3, 1 (1998)

[17] F. Abe et al. (CDF), Phys. Rev. D41, 2330 (1990)

[18] V. Khachatryan et al. (CMS), Phys. Rev. Lett. 105, 022002 (2010), 1005. 3299

[19] K. Aamodt et al. (ALICE Collaboration), Eur. Phys. J. C68, 345 (2010), 1004. 3514

[20] T. Pierog, K. Werner, Phys. Rev. Lett. 101, 171101 (2008), astro-ph/0611311

[21] M. Chojnacki (ALICE Collaboration), J. Phys. G38, 124074 (2011), 1109.6744

[22] S. Chatrchyan et al. (CMS Collaboration), Eur. Phys. J. C72, 2164 (2012), 1207 . 4724

[23] M. Ataian et al. (EHS-NA22 Collaboration), Z. Phys. C54, 247 (1992)

[24] N. Agababyan et al. (EHS-NA22 Collaboration), Z. Phys. C46, 387 (1990)

[25] N. Abgrall et al. (NA61/SHINE Collaboration), Phys. Rev. C84, 034604 (2011), 1102.0983

[26] O. Adriani et al. (LHCf Collaboration), JINST 3, S08006 (2008)

[27] H.J. Drescher, G.R. Farrar, Phys. Rev. D67, 116001 (2003), astro-ph/0212018

[28] J. Abraham et al. (Pierre Auger), Nucl. Instrum. Meth. A523, 50 (2004)

[29] H. Kawai et al. (TA), Nucl. Phys. Proc. Suppl. 175176, 221 (2008)

[30] T. Antoni et al. (KASCADE), Nucl. Instrum. Meth. A513, 490 (2003)

[31] M. Bleicher et al., J. Phys. G: Nucl. Part. Phys. 25, 1859 (1999)

[32] J. Abraham (Pierre Auger), Phys. Rev. Lett. 104, 091101 (2010), 1002.0699

[33] J. Blümer, R. Engel, J.R. Hörandel, Prog. Part. Nucl. Phys. 63, 293 (2009), 0904.0725

[34] K.H. Kampert, M. Unger, Astropart. Phys. 35, 660 (2012), 1201.0018

[35] D. Fuhrmann et al. (KASCADE-Grande Collaboration) (2013), 1308.2098

[36] A. Aab et al. (Pierre Auger Collaboration) (2013), 1307.5059 